\title{
Commentary: Patience is a virtue: Recovery is only possible if given a chance to happen, but is this safe?
}

Kyle W. Riggs, MD, and David L. S. Morales, MD

From the Department of Cardiothoracic Surgery, Cincinnati Children's Hospital Medical Center, Cincinnati, Ohio.

Disclosures: D.L.S.M is a consultant and instructor for Berlin Heart, Inc; a proctor and consultant for SynCardia, Inc, and national primary investigator for the 50/50cc SynCardia TAH Food and Drug Administration trial; a consultant for Medtronic Inc (HeartWare Division); and a consultant for Abbott Medical Inc (Thoratec Division). K.W.R has nothing to disclose with regard to commercial support.

Received for publication Dec 26, 2018; accepted for publication Dec 26, 2018; available ahead of print Jan 31, 2019.

Address for reprints: Kyle W. Riggs, MD, 3333 Burnet Ave, Cincinnati, OH 45229 (E-mail: kyle.riggs@cchmc. org).

J Thorac Cardiovasc Surg 2019;157:1618-9

$0022-5223 / \$ 36.00$

Copyright (C) 2019 by The American Association for Thoracic Surgery

https://doi.org/10.1016/j.jtcvs.2018.12.087

In their excellent single-center series review in this issue of the Journal describing the outcomes of intracorporeal centrifugal-flow ventricular assist device (VAD) support in children, Adachi and colleagues ${ }^{1}$ provide evidence for 2 extremely important concepts within the rapidly maturing field of pediatric VADs. First, they demonstrate the feasibility of consistently and safely supporting children for intermediate and long durations with home discharge. Second, they demonstrate encouraging potential for myocardial recovery in dilated cardiomyopathy with a focused protocol. In their series of 39 patients with centrifugal-flow VAD support, Adachi and colleagues ${ }^{1}$ prospectively intended to support all patients for a minimum 3 months before making decisions regarding transplantation listing. This practice, coupled with great patient selection and timing of device placement, led to a $95 \%$ home discharge rate. The waiting period was instituted to allow time for physical, functional, and psychologic recovery. What they found is that such a waiting period may also allow time for cardiac recovery in some patients with cardiomyopathy. ${ }^{2}$ Although these patients had a median of 3 adverse events each, the clustering of events in patients who died compared with no events in 10 patients is an important finding. Their outcomes demonstrate, however, that it is safe to delay transplantation to optimize candidacy and to ensure that opportunities for cardiac recovery are not missed.

Possibly the most exciting finding reported from this series of predetermined minimum duration of support with ambition for recovery in patients was that myocardial function can be restored in a considerable number of pediatric patients who would have otherwise have undergone transplantation. Also, unlike in other series, pathologic confirmation that these patients did not have myocarditis is an important point. Long-term outcomes

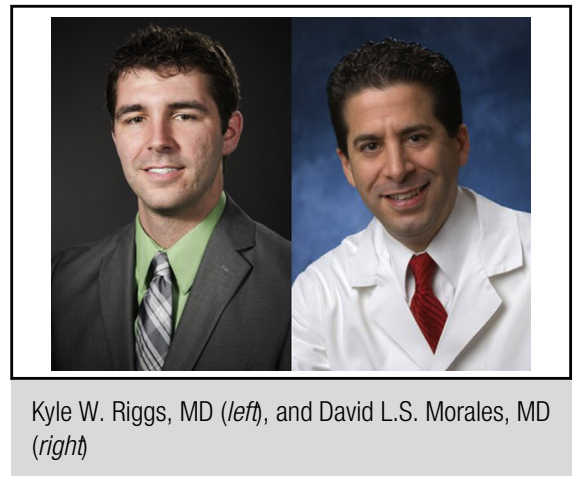

Central Message

Should VAD implantation be followed by a period of rehabilitation at home to assess pediatric patients for the potential for reversion to medically manageable outpatient heart failure? Is this safe?

See Article page 1609.

of those who recovered myocardial function and were weaned from VAD support are completely unknown, although it is clear that this recovery is almost never back to a state of no heart failure. The goal is to revert back to a level of heart failure that can be managed successfully with oral medications at home. The advantage of this is that even if heart failure again becomes unmanageable, the entire armamentarium of heart failure therapy (intravenous medical therapy, VAD, transplantation) is still available to these patients. This is demonstrated by 1 patient from the current series who was weaned from support but required subsequent VAD support and, ultimately, transplantation.

Another thought-provoking finding is their patient supported for 6.5 years. This brings up the question of VAD therapy as a long-term treatment of pediatric heart failure, as opposed to a bridge to something else. With improving hemodynamic and complication profiles, this strategy of long-term mechanical circulatory support may be less than a decade away for children. Overall, this report has given the pediatric VAD community considerable insight into the potential long-term success of implantable centrifugal-flow devices. We look forward to learning more about the patients in whom the device was explanted for recovery in the coming years. Adachi and colleagues ${ }^{1}$ 
have also demonstrated that in a mature pediatric VAD program, it is now safe to allow patience to be a virtue in our field, so that patients can gain the entire benefit from their devices: home discharge, physical and mental rehabilitation, and potential recovery—or, perhaps, even the choice to live with the device.

\section{References}

1. Adachi I, Zea-Vera R, Tunuguntla H, Denfield SW, Elias B, John R, et al. Centrifugal-flow ventricular assist device support in children: a single-center experience. J Thorac Cardiovasc Surg. 2019;157:1609-17.e2.

2. Drakos SG, Mehra MR. Clinical myocardial recovery during long-term mechanical support in advanced heart failure: insights into moving the field forward. $J$ Heart Lung Transplant. 2016;35:413-20. 